\title{
RADIOLOGY OF THE LUNG IN LEFT HEART FAILURE
}

\author{
BY
}

\section{S. SHORT*}

From the Cardiac Department, The London Hospital, and the Department of Cardiology and the Institute of Clinical Research and Experimental Medicine, The Middlesex Hospital

\author{
Received May 31, 1955
}

James Hope (1839) was probably the first to recognize that engorgement of the lungs was the cause of cardiac asthma. For almost a century, students were taught to seek for basal rales and signs of hydrothorax as evidence of such engorgement. During the past 30 years, however, as a result of the pioneer work of Laubry et al. (1929), Zdansky (1929, 1933), Kerley (1933), Parkinson (1933), and Roesler (1937), the superiority of radiological over clinical examination in this field has become recognized. Further advances in the radiology of the lung in heart disease have been made by Bedford and Lovibond (1941), Donzelot and Heim de Balsac (1947), and Jackson (1951).

Recent progress in radiological technique has greatly facilitated the study of the lung fields. It is opportune, therefore, to re-examine the changes that take place in heart disease. A previous study (Short, 1955) dealt with the pulmonary changes in severe mitral stenosis. The present investigation is concerned with the lung pattern in left heart failure.

\section{METHODS}

A consecutive series of patients undergoing investigation on account of left heart failure or left ventricular enlargement were examined radiographically. Patients were omitted from the series if there was evidence of organic disease of the mitral valve or lung parenchyma, or if the radiograph was unsatisfactory. Patients with emphysema were therefore excluded, but not those with bronchitis alone. The criteria of a satisfactory film were those laid down by the Medical Research Council X-ray Subcommittee.

Of the 51 patients admitted to the series, 31 suffered from hypertension with or without cardiac infarction; in five cases, the hypertension was of the malignant variety and in one it was due to chronic nephritis. Five had cardiac infarction without hypertension, three had left bundle-branch block of undetermined ætiology and twelve had aortic valve disease. In one, without evident heart disease, left heart failure developed during a prolonged attack of auricular tachycardia. The ages of the patients ranged from 20 to 72 , four-fifths being between 40 and 70 years old.

A full history was recorded in each case, particular attention being paid to symptoms suggestive of heart failure. In the clinical examination, special note was made of the presence of triple heart rhythm and signs of venous engorgement. Each patient had at least one electrocardiogram. One patient was in established auricular fibrillation, two suffered from paroxysmal auricular fibrillation, and one from paroxysmal auricular tachycardia. The remainder were in sinus rhythm.

Thirty-four patients were observed in 43 episodes of clinical heart failure and, either during the course of failure or subsequently, 63 radiographic examinations were made. Thirty-four radiograms were taken in the presence of heart failure, 21 within a month of the subsidence of failure, and 8 more than two months after. In 19 episodes, paroxysmal dyspnœa was the dominant symptom. Four episodes were marked by persistent orthopnœa, five by dyspnœa on exertion only, and sixteen were associated with right heart failure, as shown by jugular venous engorgement or hepatic enlargement accompanied by odema.

Fifteen patients were observed in 17 episodes of doubtful heart failure in the course of which 19 radiograms were taken. The doubt generally arose over a history of dyspnœa occurring only on exertion or attacks simulating cardiac asthma. In three patients clinical evidence of failure appeared later.

* Part of this work was done while holding a Leverhulme Research Scholarship. 
Nine patients without clinical evidence of heart failure were examined radiographically, two of them twice. Four later developed failure.

Each radiographic examination was performed in the X-ray department and consisted of a posteroanterior film taken with a tube-film distance of six feet, at $300 \mathrm{~mA}$, approximately $65 \mathrm{kV}$ and $1 / 10$ second exposure. A left lateral film was exposed in most instances, and often a right lateral also. Ward films were not requested and thus no attempt was made to follow in detail the resolution of pulmonary congestion.

The films were examined without knowledge of the history and clinical findings, particular attention being paid to the size of the pulmonary trunk, its main and its intra-pulmonary branches, the size of the pulmonary veins, the presence of hilar and pulmonary clouding, thickening or duplication of the lung fissures, and hydrothorax. The parenchymal pattern was scrutinized for interlobular lines and other abnormalities (Fig. 1).

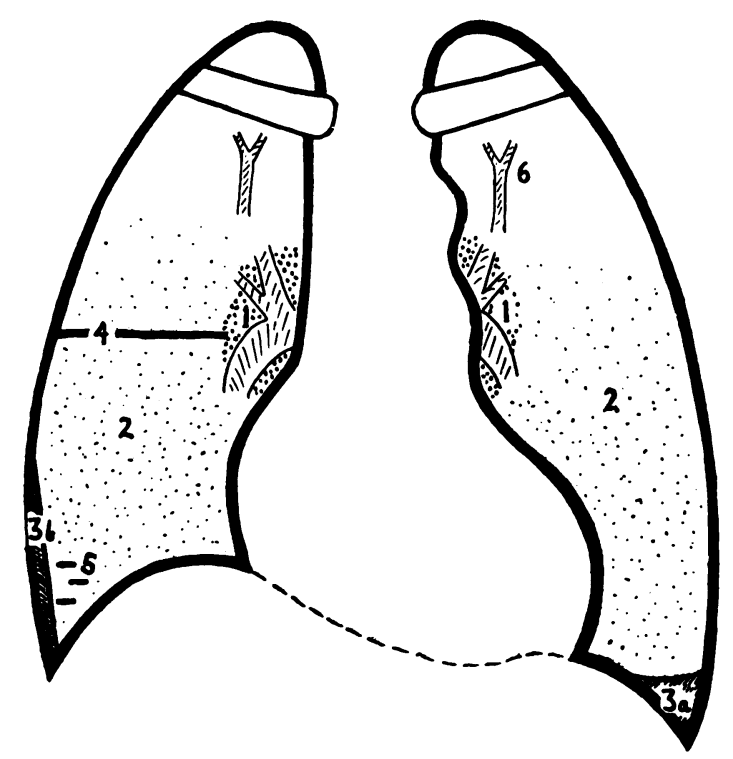

FIG. 1.-Pulmonary congestion in left heart failure. (1) Hilar clouding; (2) Pulmonary clouding; (3) Hydrothorax, (a) costo-phrenic, (b) lamellar; (4) Interlobar line;

(5) Interlobular lines; (6) Venous distension.

One of the greatest difficulties in radiographic interpretation is to determine the bounds of normality. This applies to pulmonary and especially to hilar clouding, the recognition of which leads to frequent disagreement even amongst experienced observers. Indeed, Laubry et al. (1939) have stated that the individual variability of the hilum and the anatomical and vaso-motor state of pulmonary arteries rendered the appreciation of venous stasis impossible at the outset. Advances in technique, while they have reduced the difficulty, have not eliminated it. In the present study, the dividing line has been drawn in such a way that few normal hila might be classed as abnormal though some abnormal ones might be classed as normal.

Hilar clouding, hydrothorax, interlobular lines, and indeed each feature of pulmonary congestion may be simulated more or less closely by non-cardiac diseases, so it is important to consider the lung changes in heart failure not only in detail but also as a composite picture.

\section{RESULTS}

An abnormal lung pattern was observed in 67 radiograms from 39 patients. The commonest abnormality was hilar clouding. The margins of the pulmonary arteries and their lobar branches were veiled, and the lumen of the right bronchus obscured, in 61 instances. Hilar clouding was moderate in 46 instances and considerable or great in 15 (Fig. 2 and 3). 


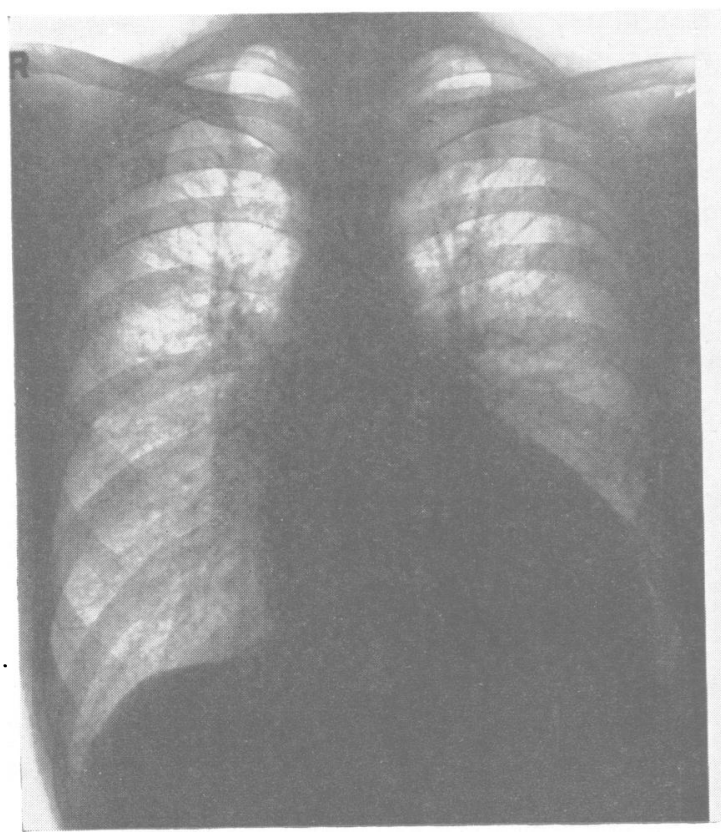

A

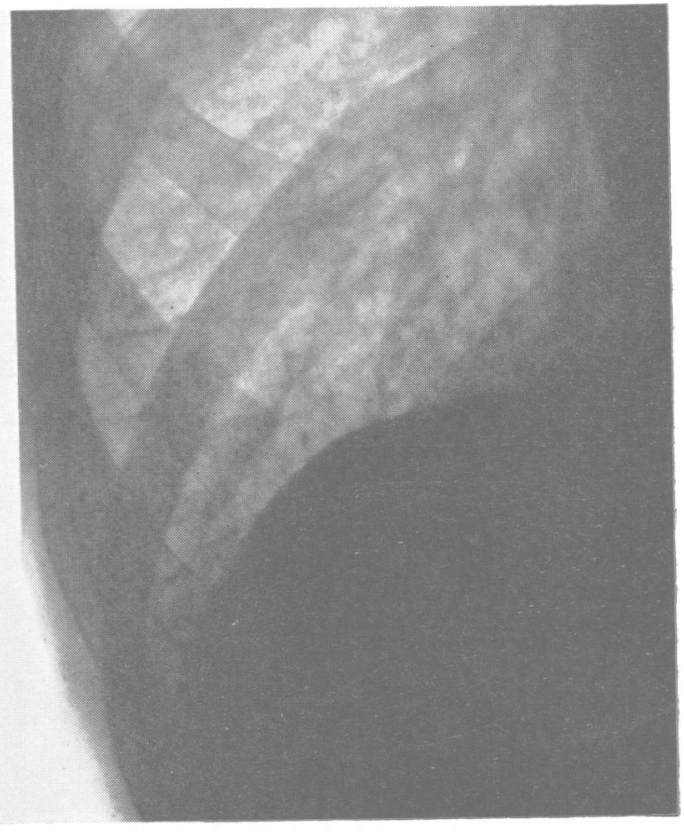

B

FIG. 2.-Left heart failure due to aortic stenosis. (A) Full chest. (B) Contact print of right lower zone. Note.-Hilar and pulmonary clouding, and interlobular lines in the right costophrenic angle.

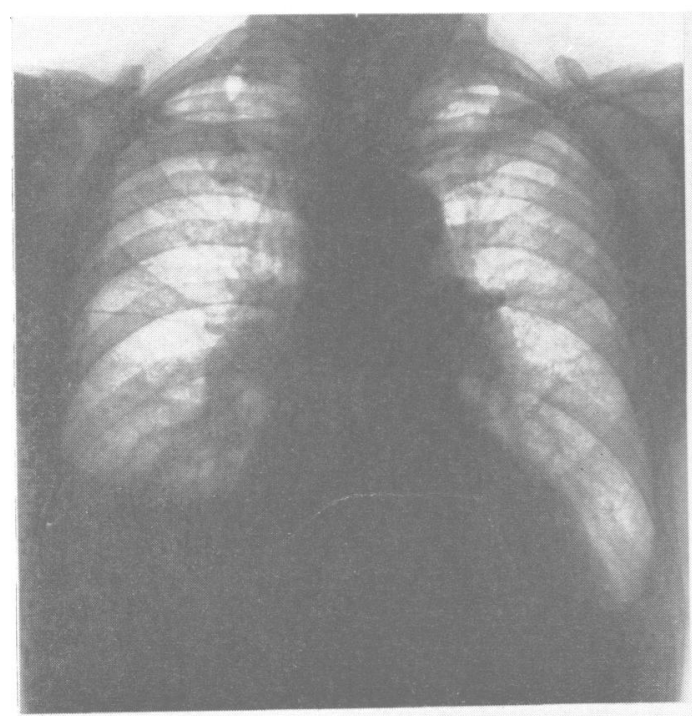

A

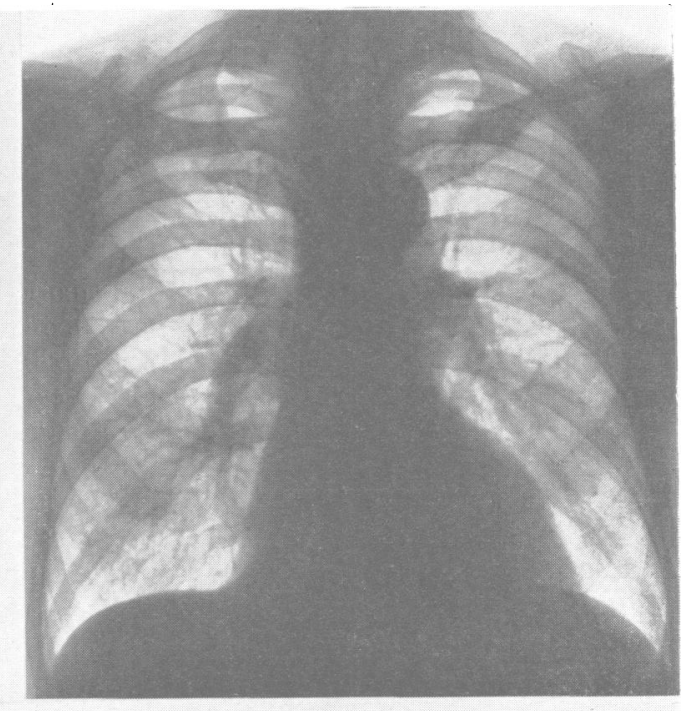

B

FIG. 3.-Biventricular failure due to hypertension. (A) At the height of failure, showing hilar clouding, interlobar line, and bilateral hydrothorax. (B) Three weeks later, showing almost complete resolution. 
Involvement of the pleura was the next most frequent finding, being observed in 51 films. Hydrothorax was present in 29 and widening of the horizontal or oblique fissures (Fig. 3 ) in the remainder. The amount of fluid varied from an effusion covering three-quarters of the hemi-thorax to a mere trace in the costophrenic sulcus, perhaps visible only in the lateral projection, or a small lamellar collection. The latter, consisting of a thin layer of fluid overlying the costal surface of the lung and visible only in a tangential projection, was seen in 4 patients. In three, the underlying lung was marked by interlobular lines (Fig. 4). The hydrothorax was bilateral in 17, right-sided in 10 and left-sided in 2 instances. Where it was bilateral the larger collection was frequently leftsided. Thus in nine cases, the greater amount was on the left, in four cases on the right, while in four, the two effusions were approximately equal.

Examination of the clinical notes of the patients with hydrothorax showed that 13 were in isolated left heart failure and 16 in biventricular failure. There was no significant difference in the localization of the effusions in the two groups.
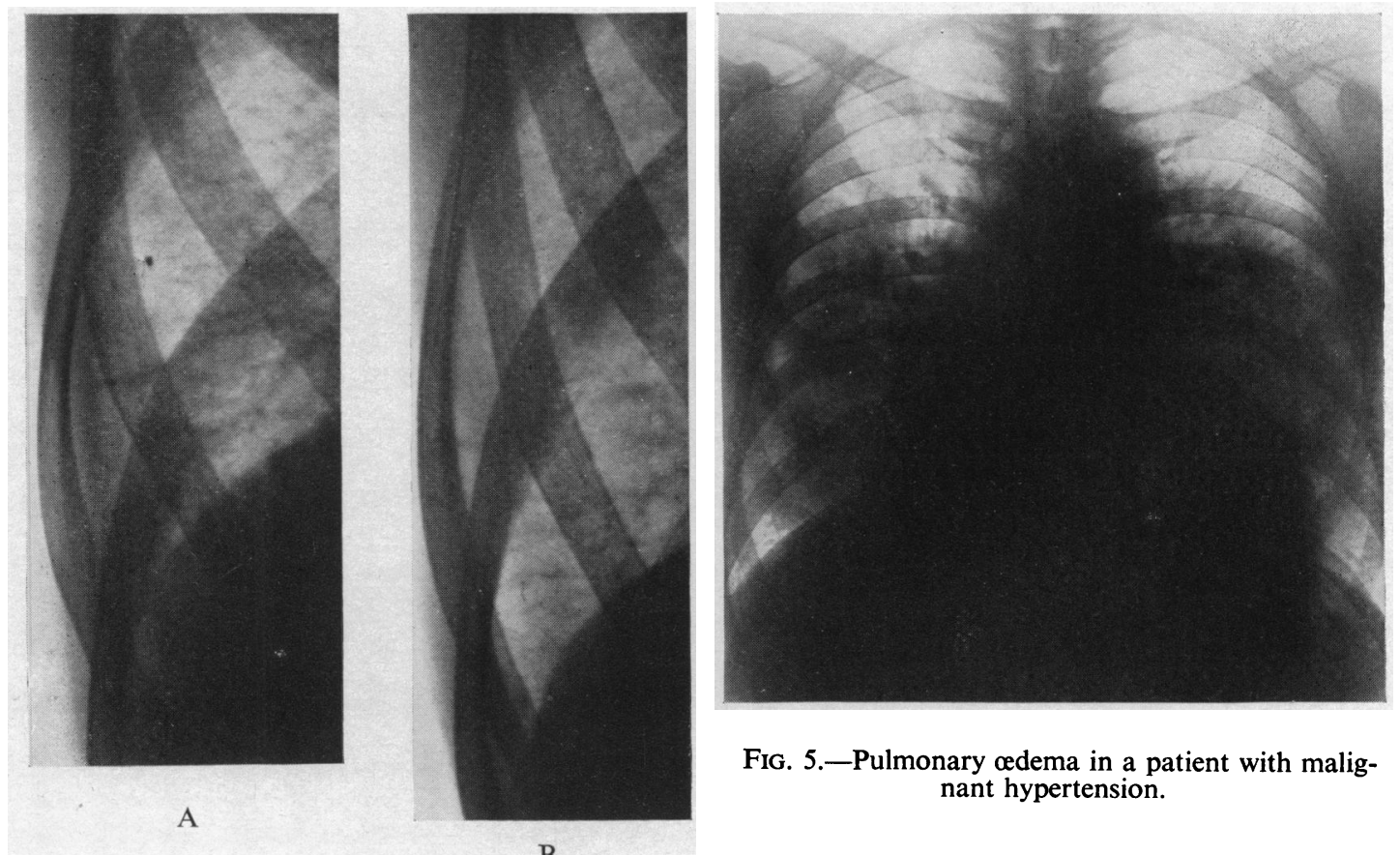

Fig. 5.-Pulmonary œdema in a patient with malignant hypertension.

FIG. 4.-Right costophrenic angle of a patient with malignant hypertension. (A) In failure, showing lamellar effusion and faint interlobular lines. (B) Two weeks later, showing effusion absorbed. The diaphragm is at a lower level in $\mathbf{B}$.

The lung translucency was reduced, particularly in the lower half, with consequent loss of definition of the ribs, heart, and pulmonary vessels in 40 films. Pulmonary clouding was generally moderate in degree and only twice did the appearance indicate frank pulmonary odema. Once both lungs were occupied by rather discrete, spherical clouds $1-2 \mathrm{~cm}$. in diameter (Fig. 5). In the second case, long streaks radiated out from the hilum like the spokes of a wheel. Widening of the oblique fissures frequently contributed to the opacity. An appearance suggesting hæmosiderosis was never seen.

Short horizontal lines were seen in the costophrenic angles in 20 films and in 10 were associated with a small effusion (Fig. 2 and 4). They were similar in appearance to the lines encountered in :severe mitral stenosis (Short, 1955), though neither so numerous nor, as a rule, so sharply defined 
as in that condition. These lines, first described by Kerley (1933), correspond to the interlobular septa. I have previously referred to them as septal lines but the term interlobular lines seems preferable.

Enlargement of the pulmonary trunk was observed in 13 radiograms and once the enlargement extended into the main branches (Fig. 3). The intrapulmonary arteries were frequently obscured, especially in the lower half of the lung, but when visible, their calibre was within normal limits. Pulmonary veins were often recognized in the upper lobes, but venous distension was conclusively demonstrated only eight times.

Clinical assessment, made independently of the radiological study, indicated present or past heart failure corresponding to 58 of the 67 films reported as abnormal. In 32 instances clinical signs of heart failure were present at the time of X-ray examination, in 19 failure had recently subsided, and 2 to 16 months had elapsed since the last episode or failure in 7 cases.

Radiograms demonstrated the presence of pulmonary congestion in one patient whose symptoms suggested isolated right heart failure.

A man, aged 67, suffering from cardiac infarction and left bundle branch-block developed swelling of the scrotum followed shortly by œdema of the legs. Dyspnœea on exertion was minimal. All signs of systemic venous congestion had subsided when he attended hospital for examination; the third heart sound was, however, audible. The radiogram showed moderate enlargement of the left ventricle, right atrium, and pulmonary artery, considerable hilar clouding, and widening of the horizontal and oblique fissures.

Pulmonary congestion was demonstrated in seven patients in whom the existence of heart failure was uncertain after careful clinical examination. Each patient denied paroxysmal dyspnœa, whether occurring spontaneously or following exertion, and admitted to only slight dyspnoea on effort. Clinical evidence of right heart failure was absent and a third heart sound was heard twice only. The following two cases illustrate this point.

A man, aged 37, with a blood pressure of 250/130 was anxious over a past syphilitic infection. He was admitted to hospital for lumbar puncture and chest X-Ray to exclude syphilitic disease of the central nervous system and aorta. In response to detailed questioning he admitted being breathless after climbing about ten stairs but said he could walk any distance on the flat. He had never been orthopnoic except once during a fog when he developed a cough. There were no signs of venous engorgement and there was no triple heart rhythm. In view of the obvious anxiety neurosis the breathlessness was discounted, and when the X-ray showed the aorta to be normal, the patient was discharged with reassurance. Inspection of the lung fields, however, showed clear evidence of pulmonary congestion, namely, hilar and pulmonary clouding, widening of the horizontal fissure, a lamellar effusion and interlobular lines. Eight months later, the patient returned with great dyspnoea and signs of venous engorgement. He had passed the stage of isolated left heart failure and entered that of biventricular failure.

A man, aged 64, suffering from syphilitic aortic incompetence and auricular fibrillation had been taking digitalis regularly for four years. Though there was considerable cardiac enlargement he had never been in failure. When he attended for routine surveillance of his syphilitic lesion, he made no complaint of dyspnoea. A radiographic examination was requested in order to determine the size of the aorta. This was found to be satisfactory, and since failure was not suspected, the patient was reassured. Later, when attention was directed to the lung fields, the presence of pulmonary congestion was evident for there was hilar and pulmonary clouding, a lamellar effusion and interlobular lines. When he next attended hospital three months later, he was complaining of paroxysmal nocturnal dyspnoea and the recent appearance of dropsy.

Fourteen patients, whose initial radiographic examination was made while in heart failure were subsequently re-examined on one or more occasions. In one patient, two episodes of heart failure were followed in this way. In nine instances, the interval between the first and second examinations was 10 to 18 days, in four 22 to 30 days, in one two months and in another nine months.

In four instances, where there was no amelioration in the patient's condition, the radiogram showed no improvement. Effusions persisted or even increased despite some decline in hilar and pulmonary clouding in two cases. In three instances, where there was moderate improvement, 
the film showed some, though incomplete resolution. Hilar and pulmonary clouding receded, but twice a small hydrothorax and once interlobular lines persisted.

In eight instances where clinical signs of failure cleared rapidly, the radiographic improvement was also striking (Fig. 3). Within 10 to 21 days, large effusions vanished, pleural thickening subsided, lung translucency returned to normal, and interlobular lines disappeared. Hilar clouding invariably decreased, though only twice did it disappear altogether. Once was in a patient whose heart appeared healthy and in whom heart failure was precipitated by tachycardia. The other was in a patient with hypertensive heart failure whose clinical recovery was remarkably complete.

In 10 instances, the radiological evidence of pulmonary congestion was equivocal. In six of these, the corresponding clinical examination was also equivocal. In two, there had been previous heart failure, one was believed actually to be in early failure, and the last showed no evidence of heart failure at that time but developed it subsequently.

Seventeen films were normal. Ten related to patients who had never been in heart failure, five to patients in whom the clinical findings were equivocal, and two to patients who had previously suffered from heart failure but had made exceptionally good recoveries.

\section{Discussion}

It is rarely practicable or necessary to conduct a radiographic examination during an attack of acute pulmonary œdema. The striking picture of intense pulmonary clouding has, however, been well described by Jackson (1951). If the attack is mild and radiographic examination is postponed for several hours the signs of pulmonary congestion may be slight or altogether absent since resolution is often both rapid and complete.

When left heart failure develops more gradually, the most striking changes in the lung fields are hilar clouding and widening of the interlobar fissures, often accompanied by effusion. Although usually bilateral or right-sided, hydrothorax is often confined to the left side, particularly in the presence of sinus rhythm and isolated left heart failure (Bedford and Lovibond, 1941).

The fact that hydrothorax may occur in isolated left heart failure was first pointed out by Bedford (1939) and is now generally recognized. Hydrothorax is indeed more characteristic of failure of the left than of the right heart. In isolated right heart failure, as in tricuspid valve disease, the infrequency of hydrothorax is in marked contrast to the constancy of ascites and dropsy. In isolated left heart failure, on the other hand, hydrothorax is common. The fact that it is more common when failure of the right heart follows defeat of the left is surely due to the fact that this represents a more advanced stage of left heart failure.

A large hydrothorax is readily detected, but small effusions are easily missed. Fluid in the posterior costophrenic sulcus may be overlooked unless the patient is rotated into the oblique or lateral position. A similar procedure is necessary in order to demonstrate fluid within the oblique fissure.

A lamellar effusion must be distinguished from the intercostal muscles which are sometimes seen tangentially as a straight or slightly convex line when the ribs overlap at a steep angle (Kerley, 1951). The distinction is, as a rule, readily made for the pulmonary surface of an effusion is usually concave, and never convex; moreover the underlying lung is often marked by interlobular lines.

Other changes in the lung fields in subacute and chronic left heart failure include pulmonary clouding with a basal preponderance and interlobular lines. The latter must not be confused with the blood vessels which are sometimes seen in the costophrenic angles in health (Short, 1955). The pulmonary artery trunk is not infrequently enlarged but distension of the veins in the upper lobes is seldom distinctive enough to be helpful.

With the subsidence of failure, pulmonary congestion abates quickly. Within a week or two the parenchyma clears, interlobular lines vanish, hilar clouding recedes, and effusions are absorbed. Nevertheless, resolution is rarely quite complete for, as Evans (1952) has indicated, some hilar clouding generally persists. The precise nature of the structural changes involved in hilar clouding is not yet known, so that the significance of their persistence is uncertain. Undoubtedly, the main 
branches of the pulmonary artery may remain dilated or unfolded after recovery and so impart to the hila an abnormal prominence (Fig. 3), but where actual clouding persists, latent heart failure must be considered likely.

Accurate detection of pulmonary congestion is best achieved by a combination of radiography with radioscopy. Radioscopy is eminently suitable for the detection of hilar clouding and pleural effusions. Radiography is superior in the demonstration of parenchymal detail and particularly of interlobular lines.

Where the existence of heart failure is uncertain after a careful clinical examination, a radiogram is frequently conclusive. In the present series, there were 17 episodes in which the clinical evidence was equivocal and doubt was resolved in all but five.

The problem commonly arises in a patient with hypertension who complains of dyspnœa only on effort and admits to none of the other symptoms of left heart failure. Other potential causes of dyspnœa, such as bronchitis or a recent increase in weight, may be evident. Physical examination reveals signs of cardiovascular hypertrophy without venous engorgement or a third heart sound. The electrocardiogram shows the pattern of left ventricular enlargement with normal $\mathbf{P}$ waves and cardioscopy shows moderate enlargement of the left ventricle and doubtful hilar clouding.

The diagnosis is not merely of academic interest for, if early heart failure is overlooked, and treatment withheld the patient may next appear in advanced failure as happened to two patients in the present series. If failure is absent, however, such treatment is unnecessary and in some respects harmful. The cause of the dyspnœa should be explained, appropriate measures advised and the patient reassured about the state of his heart. A radiographic examination is a most helpful guide to the presence of left heart failure. The demonstration of pulmonary congestion indicates failure, while its absence excludes it. There is inevitably a borderline group in which doubt remains, but it is small.

The fact that unequivocal evidence of pulmonary congestion is sometimes found in patients with very slight symptoms suggests that it may occasionally be possible to diagnose left heart failure before the appearance of any symptoms. This view is supported by the observation that hæmodynamic and anatomical changes in the lesser circulation precede symptoms.

Failure of the left ventricle leads to a rise in pressure, primarily in the left atrium, and secondarily in the pulmonary artery. In well marked left heart failure, right ventricular hypertrophy is a constant finding at necropsy (Traube, 1856), and catheterization studies have shown that pulmonary hypertension may be as severe as in mitral stenosis (Lenegre and Maurice, 1947; Lewis et al., 1953). Hypertrophy of the right ventricle (Thompson and White, 1936) and of the muscular arteries of the lung (Smith et al., 1954) are, however, sometimes found at necropsy in patients with systemic hypertension or aortic valve disease who showed no clinical evidence of heart failure and pulmonary hypertension has occasionally been demonstrated in similar groups of patients, even at rest.

Radiological evidence of pulmonary congestion should therefore be sought in all patients with severe hypertension, aortic valve disease, or cardiac infarction, even in the absence of any complaint of dyspnœa. Radiography following exercise may prove an even more sensitive test of left ventricular competence than the standard radiogram at rest.

The pattern of pulmonary congestion in left heart failure differs from that found in mitral stenosis (Short, 1955) in two important respects. In mitral stenosis, hydrothorax is infrequent and usually unilateral, whereas in left heart failure hydrothorax is common and generally bilateral. In mitral stenosis, the pulmonary changes are persistent and progressive, whereas in left heart failure they are, for the most part, transient.

There are other less striking differences between the two groups. In mitral stenosis, the boundary of the hilum is usually sharp, loss of translucency is associated with increased parenchymal detail, and hæmosiderosis is not uncommon. In left heart failure, on the other hand, the margin of the hilum is frequently blurred, parenchymal detail tends to be clouded, and hæmosiderosis is very rare: Interlobular lines are found in a high proportion of patients with severe mitral stenosis; 
they are sharply defined and frequently profuse. In left heart failure the lines are less common, less sharply defined, scanty and often associated with a small hydrothorax. In mitral stenosis the enlargement of the pulmonary trunk tends to be greater than that of its branches, whereas in left heart failure, the reverse is true.

The distinctive radiological patterns of left heart failure and mitral stenosis reflect basic hæmodynamic and pathological differences between the two diseases. One is the response of the lung to a labile pulmonary venous pressure, the other, to a persistently elevated and slowly rising pressure. One is engorgement, the other induration.

Recognition of these patterns should prove helpful in the diagnosis of rheumatic heart disease. In a patient with both mitral and aortic stenosis, for example, a mitral pattern would indicate that mitral stenosis was the dominant lesion, whereas a heart failure pattern would indicate that the aortic lesion was dominant. Similarly, in a patient in whom the mitral valve only was affected, a heart failure pattern would point to some complicating factor such as advanced myocardial disease or an important degree of mitral incompetence.

\section{SUMMARY}

Ninety-three radiographic examinations were made on 51 patients with left heart failure or left ventricular enlargement.

Forty-three episodes of clinical heart failure were studied, distinctive abnormalities being demonstrated in all but one. Hilar clouding and widening of the lung fissures, with or without hydrothorax, were the most frequent findings; pulmonary clouding and interlobular lines were less constant.

There were 17 episodes in which a diagnosis of heart failure was uncertain after careful clinical examination. Doubt was resolved by radiography in all save five, pulmonary congestion being demonstrated in seven and a normal lung architecture in five.

Pulmonary congestion was demonstrated in several patients who were almost free of symptoms. In two instances where the significance of the radiological signs was overlooked, advanced heart failure developed within a few months.

The pattern of pulmonary congestion in left heart failure is contrasted with that in severe mitral stenosis, and the application of this knowledge to the diagnosis of rheumatic heart disease is indicated.

I am indebted to Dr. William Evans, Dr. Evan Bedford, and Dr. Wallace Brigden under whose care these patients were admitted to hospital, and also to Dr. Peter Kerley, for much helpful criticism. I also acknowledge the ready co-operation of the Radiological Departments at both hospitals.

\section{REFERENCES}

Bedford, D. E. (1939). Lancet, 1, 1303.

, and Lovibond, J. L. (1941). Brit. Heart J., 3, 93.

Donzelot, E., and Heim de Balsac, H. (1947). Arch. Mal. Cour, 47, 1.

Evans, W. (1952). Cardioscopy. Butterworth Medical Publications, London.

Hope, J. (1839). A Treatise on the Diseases of the Heart and Great Vessels. 3rd ed. London.

Jackson, F. (1951). Brit. Heart J., 13, 503.

Kerley, P. (1933). Brit. med. J., 2, 594.

(1951). A Textbook of X-ray Diagnosis by British Authors. 3rd ed. H. K. Lewis, London.

Laubry, C., Chaperon, R., and Sejourne (1929). Presse méd., 37, 1653.

- Cottenot, P., Routier, D., and Heim de Balsac, R. (1939). Radiologie Clinique du Coeur et des Gros Vaisseaux. Masson et cie., Paris.

Lenegre, J., and Maurice, P. (1947). Arch. Mal. Caur, 40, 173.

Lewis, B. M., Houssay, H. E. J., Haynes, F. W., and Dexter, L. (1953). Circulation Res., $1,312$.

Parkinson, J. (1933). Brit. med. J., 2, 591.

Roesler, H. (1937). Clinical Roentgenology of the Cardiovascular System. Charles C. Thomas, Springfield, Illinois.

Short, D. S. (1955). Brit. Heart J., 17, 33.

Smith, R. C., Burchell, H. B., and Edwards, J. E. (1954). Circulation, 10, 801.

Thompson, W. P., and White, P. D. (1936). Amer. Heart J., 12, 641.

Traube, L. (1856). Über den Zusammenhaug von Herz und Nieren, Krankheiten, Berlin.

Zdansky, E. (1929). Wien. Arch. inn. Med., 18, 461.

- (1933). Röntgenpraxis, 5, 248. 